Pacific Journal of Mathematics

INTERSECTIONS OF TERMS OF POLYCENTRAL SERIES OF 


\title{
INTERSECTIONS OF TERMS OF POLYCENTRAL SERIES OF FREE GROUPS AND FREE LIE ALGEBRAS, II
}

\section{T. C. HuRLey}

\begin{abstract}
This paper investigates intersections of second terms of polycentral series of free groups and free Lie algebras and derives bases for the lower central factors of the resulting factors.
\end{abstract}

Let $G_{m}$ denote the $n$th term of the lower central series a group $G$ and $L_{m}$ the ideal in a Lie Algebra $L$ generated by of products of $m$ elements. Define $G_{m, n}=\left(G_{m}\right)_{n}$ and $L_{m, n}=\left(L_{m}\right)_{n}$. Let $F$ denote a free group and $L$ a free Lie Algebra. This paper investigates $F_{m, n} \cap F_{p, q}$ and $\boldsymbol{L}_{m, n} \cap \boldsymbol{L}_{p, q}$ and the factors $F /\left(F_{m, n} \cap F_{p, q}\right)$ and $\boldsymbol{L} /\left(\boldsymbol{L}_{m, n} \cap\right.$ $\left.\boldsymbol{L}_{p, q}\right)$. Bases for the lower central factors of $F /\left(F_{m, n} \cap F_{p, q}\right)$ and for the additive group of $\boldsymbol{L} /\left(\boldsymbol{L}_{m, n} \cap \boldsymbol{L}_{p, q}\right)$ are derived. This enables us to describe $\boldsymbol{L}_{m, n} \cap \boldsymbol{L}_{p, q}$ as a product of certain ideals in $\boldsymbol{L}$ and also to describe $F_{m, n} \cap F_{p, q}$ as a product of commutator subgroups of $F$. Some special cases of the bases have been communicated to me by M. Boral.

If $m=p$ then

$$
\begin{aligned}
F_{m, n} \cap F_{p, q} & =F_{m, n} \text { for } n \geqq q \\
& =F_{p, q} \text { for } q \geqq n .
\end{aligned}
$$

Assume without loss of generality that $m \geqq p$. Then $F_{m} \subseteq F_{p}$ and if further $n \geqq q$ then $F_{m, n} \subseteq F_{p, q}$ and hence $F_{m, n} \cap F_{p, q}=F_{m, n}$. Similar remarks of course apply for the Lie Algebra. So we shall assume in what follows that $m>p$ and $n<q$.

In [1] it is defined what is meant by saying that a basic group commutator is structurally contained in $F_{m, n}$; and what is meant by saying that a basic element is structually contained in $\boldsymbol{L}_{m, n}$ is similarly defined.

It follows from [1] Lemma 4 that the basic commutators structurally contained in both $F_{m, n}$ and in $F_{p, q}$ are contained in $F_{m, n} \cap$ $F_{p, q}$. Let $S_{r}$ denote the set of basic commutators of weight $r$ structurally contained in both $F_{m, n}$ and in $F_{p, q}$ and let $T_{r}=B_{r} \mid S_{r}$ where $B_{r}$ denotes the totality of basic commutators of weight $r$. Define $T=\bigcup_{1}^{\infty} T_{r}$. We shall also use $S_{r}, T_{r}, B_{r}$ and $T$ for the corresponding Lie elements in $\boldsymbol{L}$.

THEOREM A. 
(i) The rth lower central factor of $F /\left(F_{m, n} \cap F_{p, q}\right)$ is free abelian on the set $T_{r}$.

(ii) $T$ is an additive basis for $\boldsymbol{L} /\left(\boldsymbol{L}_{m, n} \cap \boldsymbol{L}_{p, q}\right)$.

Proof. (i ) It follows from [1] Lemma 4 that $T_{r}$ generates $F_{r}\left(F_{m, n} \cap F_{p, q}\right) / F_{r+1}\left(F_{m, n} \cap F_{p, q}\right)$ and so it is only necessary to show that $T_{r}$ is linearly independent modulo $F_{r+1}\left(F_{m, n} \cap F_{p, q}\right)$. If $r+1 \leqq$ $m n$ or $r+1 \leqq p q$ then this is clear. Hence suppose $r+1=m n+s=$ $p q+t$, for $s, t \geqq 1$.

Suppose a product $\Pi$ of elements from $T_{r}$ is contained in $F_{r+1}\left(F_{m, n} \cap F_{p, q}\right)$. Then $\Pi=a b$, where $a \in F_{r+1}$ and $b \in F_{m, n} \cap F_{p, q}$. Then by Theorem $\mathrm{C}$ of [1] $b$ is a product modulo $F(m, n ; s)$ of basic commutators structurally contained in $F_{m, n}$ and also a product modulo $F(p, q ; t)$ of basic commutators structurally contained in $F_{p, q}$. Of course $b \in F_{r}$. Now from the uniqueness modulo $F_{r+1}$ of the expression for an element as a product of basic commutators, it follows that $b$ is a product of basic commutators each of which is structurally contained in both $F_{m, n}$ and in $F_{p, q}$, i.e., $b$ is a product of elements from $S_{r}$. However, $T_{r} \cap S_{r}=\Phi$ and thus $\Pi$ induces the identity in $F_{r}\left(F_{m, n} \cap F_{p, q}\right) / F_{r+1}\left(F_{m, n} \cap F_{p, q}\right)$.

(ii) The proof for the Lie Algebra case is similar and is omitted. (It also follows from part (i) by setting up a homomorphism (which is consequently an isomorphism) from $\boldsymbol{L} /\left(\boldsymbol{L}_{m, n} \cap \boldsymbol{L}_{p, q}\right)$ to the Lie Algebra formed from the direct sum of the lower central factors of $F /\left(F_{m, n} \cap F_{p, q}\right)$.)

$$
\begin{aligned}
& \text { Now define (for } m \geqq p, q>n), \\
& F(m, 1 ; p, q)=F_{m} \cap F_{p, q}, \\
& F(m, n ; p, q)=\Pi\left[F_{m} \cap F_{p, i_{1}}, F_{m} \cap F_{p, i_{2}}, \cdots, F_{m} \cap F_{p, i_{n}}\right] \text {, for } n>1,
\end{aligned}
$$

where the product is over all positive integers $i_{1}, i_{2}, \cdots, i_{n}$ such that $i_{1}+i_{2}+\cdots+i_{n}=q$.

Note that it is easily verified that $\left[F_{m} \cap F_{p, j_{1}}, F_{m} \cap F_{p, j_{2}}, \cdots, F_{m} \cap\right.$ $\left.F_{p, j_{n}}\right] \leqq F(m, n ; p, q)$ for $j_{1}+j_{2}+\cdots+j_{n} \geqq q$ and hence in the definition we could have taken $i_{1}, i_{2}, \cdots, i_{n}$ such that $i_{1}+i_{2}+\cdots+$ $i_{n} \geqq q$. In [1] Theorem A, $F_{m} \cap F_{p, s}$ is identified as a product of certain commutator subgroups of $F$. If $p s \geqq m$ then $F_{m} \cap F_{p, s}=$ $F_{p, s}$; for $m=p s+r$ the notation $F(p, s ; r)$ is used for the product of commutator subgroups which is identified with $F_{m} \cap F_{p, s}$. In [1] "structurally contained in $F_{m} \cap F_{p, s}$ " has been defined and we wish to extend this definition to defining what is meant by saying 
that a basic commutator is structurally contained in $F(m, n ; p, q)$. If $n=1$, then $F(m, 1 ; p, q)=F_{m} \cap F_{p, q}$ and structurally contained in $F_{m} \cap F_{p, q}$ has been defined. Assume $n>1$ and suppose it has been defined what is meant by saying that a basic commutator is structurally contained in $F(m, k ; p, q)$ for all $k, 1 \leqq k<n$, and for all $m, p, q$ with $m \geqq p$. If $q \leqq n$ say a basic commutator $a$ is structurally contained in $F(m, n ; p, q)\left(=F_{m, n}\right)$ iff $a$ is structurally contained in $F_{m, n}$. If $q>n$ (this forces $q \geqq 2$ ) say the basic commutator $a$ is structurally contained in $F(m, n ; p, q)$ iff $a=[b, c]$ for basic commutators $b, c$ with $b$ structurally contained in $F\left(m, n_{1}, p, q_{1}\right)$, $c$ structurally contained in $F\left(m, n_{2} ; p, q_{2}\right)$, for positive integers $n_{1}, n_{2}, q_{1}, q_{2}$ satisfying $n_{1}+n_{2}=n$ and $q_{1}+q_{2}=q$.

For a basic commutator $a$, use $a \bar{\epsilon} F(m, n ; p, q)$ to mean that $a$ is structurally contained in $F(m, n ; p, q)$.

LEMMA 1.

(i) $\quad F(m, n ; p, q) \leqq F_{m, n} \cap F_{p, q}$.

(ii) If $a \bar{\epsilon} F(m, n ; p, q)$ then $a \in F(m, n ; p, q)$.

Proof. This follows easily from e.g., [1], Lemma 1.

Proposition B. $a \bar{\epsilon} F_{m, n}$ and $a \bar{\epsilon} F_{p, q}$ if and only if $a \bar{\epsilon} F(m, n ; p, q)$.

Proof. If $a \bar{\epsilon} F(m, n ; p, q)$ then it follows easily from the definitions that $a \bar{\epsilon} F_{m, n}$ and $a \bar{\epsilon} F_{p, q}$.

Suppose on the other hand, $a \bar{\epsilon} F_{m, n}$ and $a \bar{\epsilon} F_{p, q}$. We can assume that $m>p$ and $n \leqq q$. If $p=1$ or $n=1$ there is nothing to be shown. Hence we can assume $p>1$ and $n>1$. Therefore $a=[b, c]$ with $b, c$ basic commutators and satisfying

$$
\begin{aligned}
& b \bar{\epsilon} F_{m, k_{1}}, c \bar{\epsilon} F_{m, k_{2}} \text { with } k_{1}+k_{2}=n \\
& b \bar{\epsilon} F_{p, j_{1}}, c \bar{\epsilon} F_{p, j_{2}} \text { with } j_{1}+j_{2}=q
\end{aligned}
$$

((1) follows since $n>1$ and $a \bar{\epsilon} F_{m, n}$. $\left.a \bar{\epsilon} F_{p, q}.\right)$

(2) follows since $q>1$ and

Hence, by induction, $b \bar{\epsilon} F\left(m, k_{1} ; p, j_{1}\right), c \bar{\epsilon} F\left(m, k_{2} ; p, j_{2}\right)$ giving that $a \bar{\epsilon} F(m, n ; p, q)$.

Let $a \in F_{m, n} \cap F_{p, q}$. Then from Theorem A (i), for any $r, a$ is a product modulo $F_{r+1}$ of basic commutators of weight $\leqq r$ each of which is structurally contained in both $F_{m, n}$ and in $F_{p, q}$. Hence for any $r, a=b_{r} c_{r}$ for $b_{r} \in F(m, n ; p, q)$ and $c_{r} \in F_{r+1}$. Thus up to a residual 
part $a \in F(m, n ; p, q)$. More specifically let $R=\bigcap_{i=1}^{\infty} F(m, n ; p, q) F_{i}$ and then $a \in F(m, n ; p, q) R$. Also $R \subseteq F_{m, n} \cap F_{p, q}$, since both $F / F_{m, n}$ and $F / F_{p, q}$ are residually nilpotent. I have proved

Proposition C.

$$
F_{m, n} \cap F_{p, q}=F(m, n ; p, q) R .
$$

If it could be shown that $F / F(m, n ; p, q)$ is residually nilpotent then it would follow that $\bigcap_{i}^{\infty} F(m, n ; p, q) F_{i}=F(m, n ; p, q)$. Then $F_{m, n} \cap F_{p, q}$ would be identified with $F(m, n ; p, q)$.

This is no residual problem for the Lie Algebra case, and so I get the following proposition. $(L(m, n ; p, q)$ is defined by analogy to the group case.)

Proposition D.

$$
\boldsymbol{L}_{m, n} \cap \boldsymbol{L}_{p, q}=\boldsymbol{L}(m, n ; p, q) .
$$

Proof. Suppose $a \in \boldsymbol{L}_{m, n} \cap \boldsymbol{L}_{p, q}$. Then as above we get that for any $r, a=b_{r}+c_{r}$ with $b_{r} \in \boldsymbol{L}(m, n ; p, q)$ and $c_{r} \in \boldsymbol{L}_{r}$. If $a_{j}$ denotes the $j$ th homogeneous part of $a$ then

$$
a=a_{0}+a_{1}+\cdots+a_{s} \quad(s<\infty) .
$$

Therefore $c_{s+1}=0$ giving that $a \in \boldsymbol{L}(m, n ; p, q)$.

\section{REFERENCES}

1. T. C. Hurley, Intersections of terms of polycentral series of free groups and free Lie Algebras, Pacific J. Math., 82 (1979), 105-116.

Received November 15, 1978.

UNIVERSITY COLLEge

Belfield, Doblin 4, IRELAND 


\section{PACIFIC JOURNAL OF MATHEMATICS}

\section{EDITORS}

DONALD BABBITT (Managing Editor)

University of California

Los Angeles, California 90024

Hugo RossI

University of Utah

Salt Lake City, UT 84112

C. C. MOORE and ANDREW OGG

University of California

Berkeley, CA 94720

\section{J. DugundJI}

Department of Mathematics University of Southern California Los Angeles, California 90007

R. FinN and J. Milgram Stanford University Stanford, California 94305

\section{ASSOCIATE EDITORS}
R. ARENS
E. F. BECKENBACH
B. H. Neumann
F. WOLF
K. YoshidA

\section{SUPPORTING INSTITUTIONS}

UNIVERSITY OF ARIZONA

UNIVERSITY OF BRITISH COLUMBIA

CALIFORNIA INSTITUTE OF TECHNOLOGY

UNIVERSITY OF CALIFORNIA

MONTANA STATE UNIVERSITY

UNIVERSITY OF NEVADA, RENO

NEW MEXICO STATE UNIVERSITY

OREGON STATE UNIVERSITY
UNIVERSITY OF OREGON

UNIVERSITY OF SOUTHERN CALIFORNIA

STANFORD UNIVERSITY

UNIVERSITY OF HAWAII

UNIVERSITY OF TOKYO

UNIVERSITY OF UTAH

WASHINGTON STATE UNIVERSITY

UNIVERSITY OF WASHINGTON 


\section{Pacific Journal of Mathematics}

\section{Vol. 96, No. $1 \quad$ November, 1981}

Hédi Amara, Groupe des classes et unité fondamentale des extensions quadratiques relatives à un corps quadratique imaginaire principal $\ldots \ldots \ldots 1$

Douglas S. Bridges, On the isolation of zeroes of an analytic function $\ldots \ldots 13$ Andrew J. Casson and John L. Harer, Some homology lens spaces which

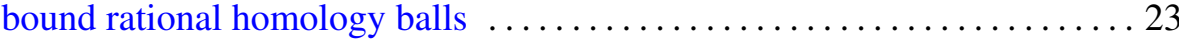

Z. A. Chanturia, On the absolute convergence of Fourier series of the classes $H^{\omega} \cap V[v]$

J.-F. Colombeau and Mário Carvalho Matos, On some spaces of entire functions defined on infinite-dimensional spaces $\ldots \ldots \ldots \ldots \ldots \ldots \ldots 63$

Edwin Duda, Pointwise periodic homeomorphisms on chainable continua . . .77

Richard F. Gustafson, A simple genus one knot with incompressible spanning surfaces of arbitrarily high genus $\ldots \ldots \ldots \ldots \ldots \ldots \ldots \ldots 1$

Fumio Hiai, Masanori Ohya and Makoto Tsukada, Sufficiency, KMS condition and relative entropy in von Neumann algebras

Ted Hurley, Intersections of terms of polycentral series of free groups and free Lie algebras. II .................................. 111

Robert Edward Jamison, II, Partition numbers for trees and ordered sets . . 115 R. D. Ketkar and N. Vanaja, A note on FR-perfect modules ............. 141 Michihiko Kikkawa, On Killing-Ricci forms of Lie triple algebras ....... 153

Jorge Lewowicz, Invariant manifolds for regular points 163

Richard W. Marsh, William H. Mills, Robert L. Ward, Howard Rumsey and Lloyd Richard Welch, Round trinomials .....

Claude Schochet, Topological methods for $C^{*}$-algebras. I. Spectral sequences

Yong Sian So, Polynomial near-fields?

Douglas Wayne Townsend, Imaginary values of meromorphic functions in the disk

Kiyoshi Watanabe, Coverings of a projective algebraic manifold .. 243

Martin Michael Zuckerman, Choosing $l$-element subsets of $n$-element sets 